To combat television headache it is recommended that "children should sit not less than 6 feet away from the screen, which should be a little above eye level, and as nearly as possible directly in front. A low light should be kept on, placed behind, or to the side of the viewers, to reduce the glare produced which is so tiring to the eyes".

\section{THE MAGNETIC MOMENT OF THE NEUTRON}

A

NEW measurement of the magnetic moment of the neutron to a greater precision than before has recently been performed by V. W. Cohen, N. R. Corngold and N. F'. Ramsey (Phys. Rev., 104, $283 ; 1956)$. Of the simpler nuclei and nucleons, the neutron is the one the magnetic moment of which has been measured with least precision. The general method used in the past by Bloch and co-workers employed the reorientation of the moments in a beam of polarized neutrons by an alternating mag. netic field in resonance with the Larmor frequency of precession of the neutrons in a superimposed uniform magnetic field, but the accuracy was limited by the degree of polarization of the neutrons attainable by transmission through magnetized iron, the low neutron flux and the short path of the neutrons in the magnetic field.

In 1950, N. F. Ramsey showed (Phys. Rev., 78, 695) that the separated oscillatory field method of observation of resonance in a beam of magnetic particles produces a very narrow resonance line, thus permitting a precise measurement of the position of its centre, and in the following year D. J. Hughes and M. T. Burgy (Phys. Rev., 81, 498; 1951) discovered that neutrons reflected from cobalt mirrors magnetized to saturation are polarized to well over 90 per cent. These new developments have been incorporated in Cohen, Corngold and Ramsey's apparatus. A beam of slow neutrons obtained from the Brookhaven National Laboratory reactor was polarized by reflexion from a magnetized cobalt mirror and then reflected from a similar mirror used as an analyser. Between the two mirrors the neutrons pass through a region of uniform magnetic field where they may be depolarized by a resonant radiofrequency magnetic field, thus resulting in a drop in intensity of the neutrons reflected from the second mirror. The resonant frequency for depolarization is compared with the proton moment resonant fre. quency in the same transition region. For high resolution of the neutron resonance the path-length of the neutrons in the uniform magnetic field was made $110 \mathrm{~cm}$.

Full details of the apparatus together with the experimental procedure and the results obtained are given. The measured ratio of neutron and proton resonant frequencies was $v_{n} / v_{p}=0.685057 \pm$ 0.000017 , which may be compared with the last published value by Bloch of $0.685001 \pm 0 \cdot 000030$. This leads to a value for the neutron magnetic moment of $\mu_{n}=-1.913148 \pm 0.000066$ nuclear magnetons. It is clear from the discussion that the neutron resonance measurement is more precise than that for the proton; some indication is also given of how, with improvement of technique, the ratio of neutron to proton moment may eventually be measured with an accuracy better than one part in a million.

\section{MICROCLIMATE OF THE AFRICAN SAVANNA}

THE literature on the climate of the lowest layers of the atmosphere in the tropical grassland regions is small. A notable contribution has, however, been made in the publication of the report "Exploration du Parc National de la Garamba"* by A. Noirfalise, of the Institut des Pares Nationaux du Congo Belge. The area described lies in the extreme north-east of the Belgian Congo in latitude $4^{\circ} \mathrm{N}$. on the border of the Sudan. It is in the savanna country of extensive grasslands varied by clumps of bush and scattered trees, with forests of larger trees along the river valleys. The paper describes first the macroclimate. The months from December to February constitute the dry hot season. There are rainfall maxima in May and August as the sun passes over, but the intervening dip in the rainfall variation is not very pronounced. In the dry season, temperature in the screen rises to $35^{\circ} \mathrm{C}$. by day and falls to $15^{\circ} \mathrm{C}$. at night, while in the cloudy rainy season the corresponding temperatures are $23^{\circ} \mathrm{C}$. and $17^{\circ} \mathrm{C}$. The relative humidity falls below 30 per cent at midday in the dry season, but to only about 60 per cent in the wet season. During the night it is more than 80 per cent throughout the year.

The most interesting part of the book deals with the microclimate of the great grasslands. In the first half of the rainy season when the grass has a height of 40-90 $\mathrm{cm}$. the presence of the grass makes little difference to the temperature of the surface under the midday sun. 'The temperature at midday increases slowly downwards to about $5 \mathrm{~cm}$. above the soil where the temperature is about 2 deg. C. above screen temperature. Between $5 \mathrm{~cm}$. and the surface, temperature rises by about 10 deg. C. to give surface maxima of about $42^{\circ} \mathrm{C}$. This is the same as the temperature reached by a grass-covered surface on a clear midsummer day in England. As the rainy season goes on, the grass grows more thickly and exercises a pronounced shading effect on the soil. This is illustrated by comparative curves of the vertical distribution of temperature over a bare and a grassy surface on a clear day in August 1952. Over the bare surface the distribution was as described before, with a surface maximum of $45^{\circ} \mathrm{C}$. On the other hand, over the surface covered by thick grass the maximum temperature, $32^{\circ} \mathrm{C}$., occurred at $10 \mathrm{~cm}$. above the surface and the surface temperature was only about $27^{\circ}$ C. There are large patches, ancient termitiaries now levelled, of bare soil in the savanna and the extremely high temperature which they reach tends to prevent the growth of grass over them. The thick grass of the rainy season has also a strong sheltering effect on the surface during rainfall, as the catch of a gauge at $20 \mathrm{~cm}$. is reduced by some 60 per cent from that of a gauge clear of grass.

The paper also describes the microclimate under clumps of shrubs and trees in the savanna and in the forests which still exist along the rivers. The vege. tation produces a smoothing of the diurnal variation of temperature in proportion to its density. Under the dense riverside forests there is very little variation indeed.

* Institut des Parcs Nationaux du Congo Belge Ifxploration du Parc National de la Garamba. Fascicule 6: Le Milieu Climatique. aux du Congo Belge, 1956.) 